\title{
Experimental study of dynamic fragmentation of shock- loaded metals below and above melting
}

\author{
L. Signor ${ }^{1,2, a}$, E. Lescoute ${ }^{1}$, D. Loison ${ }^{1}$, T. De Rességuier ${ }^{1}$, A. Dragon ${ }^{1}$, and G. Roy ${ }^{2}$ \\ ${ }^{1}$ Institut Pprime, ENSMA - CNRS - Université de Poitiers, 86961 Futuroscope, France \\ ${ }^{2}$ CEA, Centre de Valduc, 21120 Is-sur-Tille, France
}

\begin{abstract}
The breakout and reflection of a strong shock-wave upon the free surface of a metallic sample may lead to ejecta production of many types. Spall fracture is due to tensile stresses which result from the interaction of the incident and the reflected release waves. When the sample remains in solid state, one or several layers of finite thickness, called spalls, can be created and ejected. When melting is initiated during shock-wave propagation, tensile stresses are generated in a liquid medium and lead to the creation of an expanding cloud of liquid debris. This phenomenon, sometimes referred to as microspalling, consists in a dynamic fragmentation process in the melted material. The present paper is devoted to the experimental investigation of the transition from spall fracture in solid state to the micro-spalling process in molten metals. This study, realized on tin and on iron, involves different shock generators (gas gun, pulsed laser...) and diagnostics (velocimetry, high-speed optical shadowgraphy, fragments recovery).
\end{abstract}

\section{Introduction}

When a triangular shock-wave reflects at the free surface of a metal sample, the interaction of the reflected and the incident release waves gives rise to tensile stresses. This may lead to the separation and the ejection of one or several layers of materials, called spalls (Fig. 1a). While extensive work has been dedicated to spall fracture in solid materials [1], little data can be found yet about how such process evolves when material is previously melted partially or fully, upon compression or release, during the propagation of the incident pulse (Fig. 1b). Then, tensile stresses are generated in a liquid medium and lead to the formation of an expanding cloud of molten droplets (Fig. 1c). This phenomenon, referred to as micro-spalling [2], has been evidenced in tin submitted to explosive detonation [3], laser-driven shock [4,5] and plate impact [6].

The present paper is devoted to the experimental investigation of such dynamic fragmentation phenomena in shock-loaded metals. This work includes (i) plate impact experiments using either gas or powder gun as well as (ii) laser shock experiments which notably favour the recovery of samples and fragments and allow to achieve melting in others metals such as iron. Experimental set-up is described in Section 2, then results are presented in Section 3. In conclusion, modelling and simulation issues are briefly addressed with regard to this experimental study.

\footnotetext{
a e-mail : loic.signor@1mpm.ensma.fr
} 
a.)

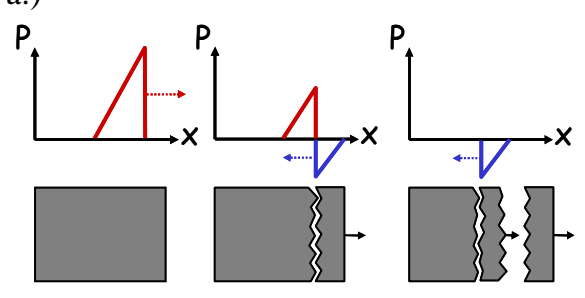

b.)

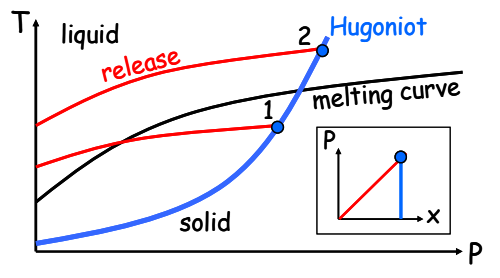

c.)
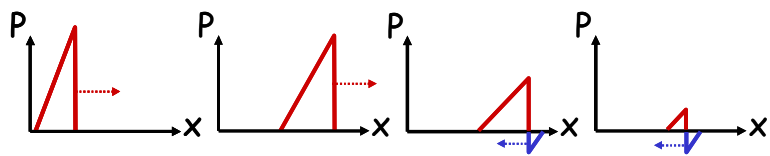

liq solid
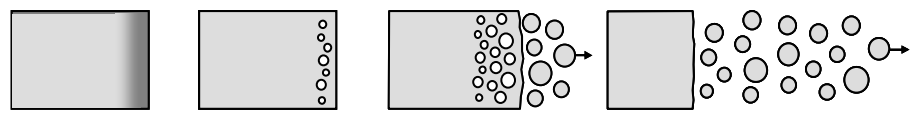

Fig. 1 a.) Schematic illustration of spallation in solids. b.) Schematic pressure-temperature phase diagram including the shock-compressed states (located on the Hugoniot curve) and subsequent release paths; for sufficiently high shock pressure, shock-induced melting may occur either on release (1) or on compression (2). c.) Schematic illustration of micro-spalling in shock-melted materials.

\section{Experimental set-up}

In the first series of experiments, a shock-wave is produced by the impact of a projectile on a target assembly composed by a copper transmitter and the tin sample (Fig. 2a). Thicknesses of flyers and targets are chosen to ensure the evolution of initially square pressure pulse into an unsupported (triangular) shock-wave propagating in tin sample.

a.)

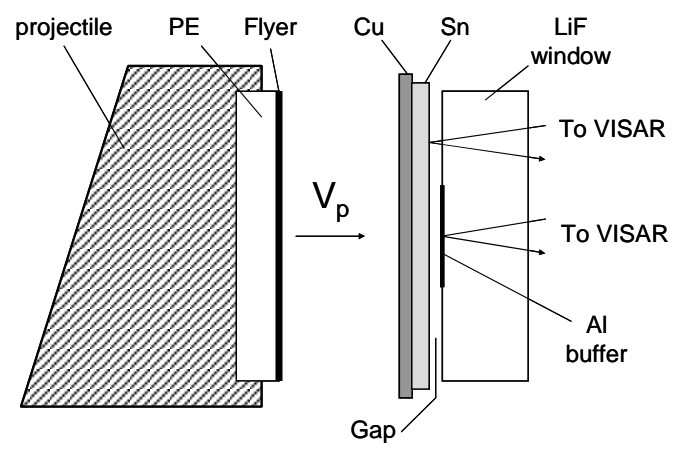

b.)

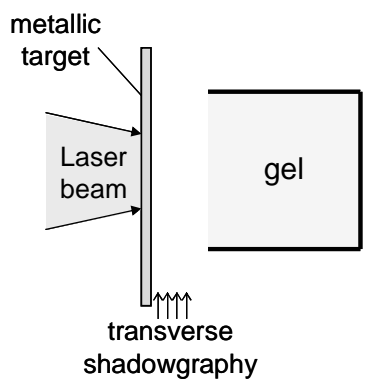

Fig. 2 Schematic of the experiments: plate impact (a), laser shock (b)

Three tests are discussed in the following. The first (Test 1) and second (Test 2) ones have been performed with Denal (Tungsten alloy) flyer accelerated at a velocity $\mathrm{V}_{\mathrm{p}}$ using a single stage powder gun. The third one (test 3 ) has been performed using a double stage gas gun and Tantalum flyer. The velocity of the free surface of tin sample (opposite to the impact plane) is measured using Velocity Interferometer System for Any Reflector (VISAR). The so-called Asay-window technique [6,7] - or thick-plate technique [8] - is employed to infer some kinematical properties of the debris cloud 
ejected from the tin free surface; velocity of the surface of the Lithium Fluoride (LiF) window impacted by tin ejecta is measured using VISAR.

According to preliminary one-dimensional (1D) Lagrangian simulations using a multiphase equation of state for tin [9], the peak shock breakout pressure in tin $\mathrm{p}_{\mathrm{s}}$ and melting occurrence have been evaluated (Fig. 3a): tin should remain in solid state during Test $1\left(p_{s}=18 \mathrm{GPa}\right.$, point 1 in Fig. 3a), then melting is achieved on release (Test $2, \mathrm{p}_{\mathrm{s}}=35 \mathrm{GPa}$, point 2 in Fig. $3 \mathrm{a}$ ) or on compression (Test 3, $\mathrm{p}_{\mathrm{s}}=68 \mathrm{GPa}$, point 3 in Fig. 3a).

(a)

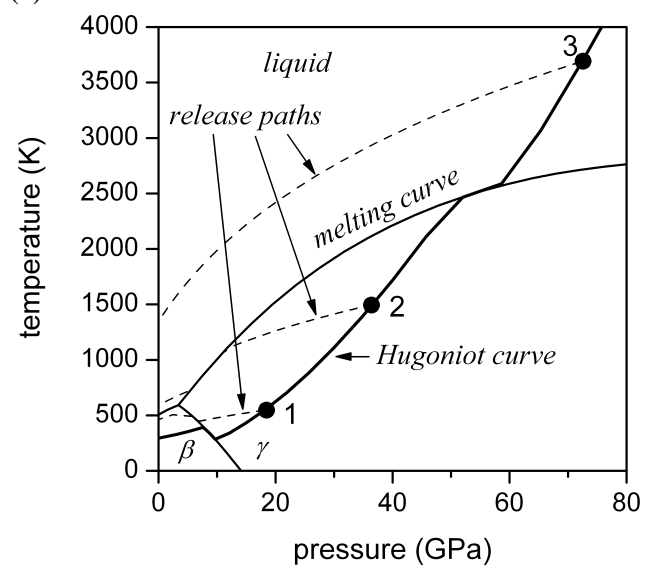

(b)

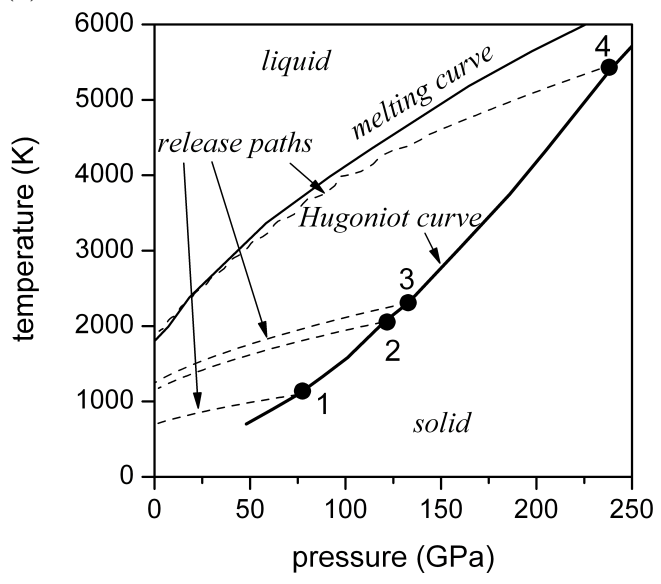

Fig. 3 Pressure-Temperature phase diagram of tin (a) and iron (b) including predicted shock states (on the theoretical Hugoniot) and subsequent release paths. In (a), points 1, 2 and 3 correspond to plate impact Tests 1, 2 and 3 in tin, respectively. In (b) are reported the data of laser-shock experiments on iron (points 1 and 2 are related to Test 4 whereas points 3 and 4 correspond to Test 5).

The second series of experiments (referred to as laser-shock experiments as schematically depicted in Fig. 2b) have been performed on iron and tin in the Laboratoire pour l'Utilisation des Lasers Intenses (LULI). A pulsed laser beam of $1.06 \mu \mathrm{m}$ wavelength, 3 to $5 \mathrm{~ns}$ duration and $\sim 5 \mathrm{~J}$ maximum energy is focused onto a metallic foil, set in a vacuum chamber to avoid laser breakdown in air. The irradiation produces the vaporization of a thin ( $\mu \mathrm{m}$-order) layer of material, transformed into a plasma cloud which expands toward the laser source and drives by reaction a compressive pulse into the solid target. During propagation through the sample, the compression front gets steeper and the release wave spreads so the pressure profile becomes roughly triangular and the peak pressure decays. Information regarding the fragmentation process is provided by means of : (i) scanned electron micrographs of recovered target, (ii) optical transverse shadowgraphs of the fragments ejected from the sample free surface and (iii) optical micrographs of fragments recovered in a low-density gel (further details of these techniques are given in [10]).

Preliminary 1D computations of the laser-matter interaction and shock-wave propagation - using the code CHIC of the Centre Lasers Intenses et Applications (CELIA, UMR5707, France) and Bushman-Lomonosov-Fortov iron equation of state [11] - have been performed to estimate the peak pressure near the spalled or micro-spalled regions, as well as the residual state of metal samples (Fig. $3 b)$. The present discussion focuses on two significant experiments on $100 \mu \mathrm{m}$-thick iron targets. For the first one (Test 4), iron remains in solid state: shock pressure $\mathrm{p}_{\mathrm{s}}$ decays from 120GPa near the irradiated surface (point 2 in Fig. 3b) to $75 \mathrm{GPa}$ beneath the free surface (point 1 in Fig. 3b). In the second one (Test 5) melting is expected to occur within one region of the target since shock pressure decays from $240 \mathrm{GPa}$ from the irradiated surface (point 4 in Fig. $3 \mathrm{~b}$ ) to $130 \mathrm{GPa}$ beneath the free

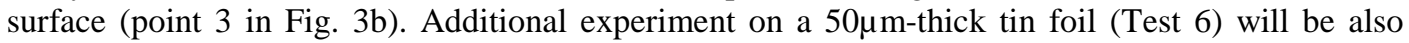
addressed in regard to the observation of fragments collected in gel. In this case, the peak shock 
pressure decays from about 150GPa near the irradiated surface to about 120GPa beneath the free surface so that the whole target thickness is fully melted on compression. For this test, experimental conditions are almost identical to those from a previous shot [12] where tin fragments were collected in PVC-foam (instead of gel) and observed by X-Ray micro-tomography (instead of optical microscopy).

\section{Results}

\subsection{Plate impact experiments}

Fig. 4 shows the velocity profiles measured during plate impact experiments on tin. A detailed analysis of such data lies beyond the scope of the present paper, but the main salient conclusions are briefly reported in the following. For Test 1 , the first part of the curve, related to the velocity of the tin free surface, exhibits the well-known spall pulse [1] which indicates the occurence of a spallation process in solid state. Then, the velocity profile measured at the window surface (see vertical arrow in Fig. 4) evidences the impact of a thick layer of material, i.e. the first spall, then possibly a second one.

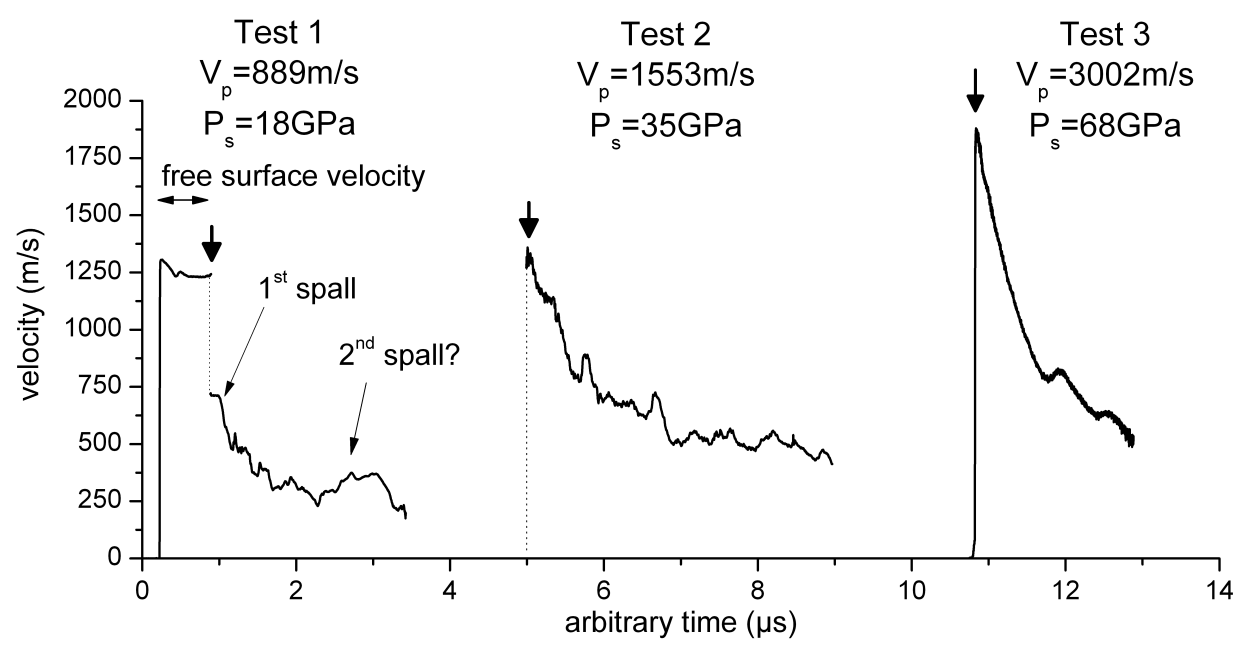

Fig. 4. VISAR velocity measurements during the three plate impact experiments on tin. The first impact of ejecta on $\mathrm{LiF}$ window is indicated for each test by the large vertical arrows.

Distinctive velocity data, obtained when tin is melted on release (Test 2) or on compression (Test 3 ), can be related the micro-spalling phenomenon. Tin free surface cannot be measured due to a sudden drop of reflectivity noticed at shock breakout which can be attributed to melting and ejection of fine droplets. In both cases, overall tendency of velocity profiles shows that the LiF window is impacted by successive layers of decreasing velocity. Such observations are consistent with the impact of a low-density medium in expansional flight as expected for the micro-spalled cloud. Furthermore, smoother profile observed for Test 3 seems to indicate a decrease of the characteristic length scale of heterogeneities within the micro-spalled debris cloud for increasing shock pressure, i.e. increasing level of melting and final temperature. However, such measurements cannot capture quantitatively the actual fine-scale structure (fragments size, shape, etc...) of the micro-spalled debris cloud. 


\subsection{Laser-shock experiments}

Optical transverse shadowgraphy provides quasi-instantaneous image of the fragments ejected from the sample free surface at controlled delay times after laser shot. Figs. 5a and 5b show such shadowgraphs recorded one microsecond after the laser shot for Test 4 and Test 5, respectively. Both pictures are consistent with the formation and the expansion of wide debris clouds made of numerous fragments. They illustrate the increase of maximal ejection velocity with shock pressure (up to $\sim 3 \mathrm{~km} / \mathrm{s}$ for Test 5 ). For Test 4 , the planarity of the front of the debris cloud seems to indicate that the loading pressure applied onto the irradiated surface is fairly homogenous. This point is more questionable for Test 5 .

a.)

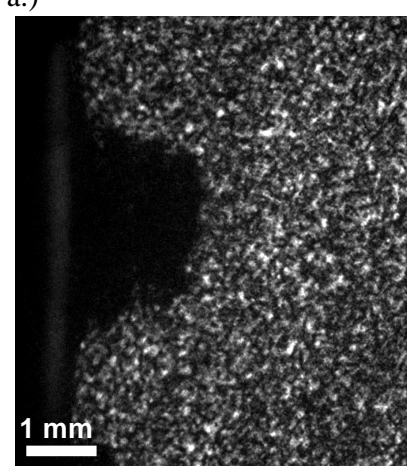

b.)

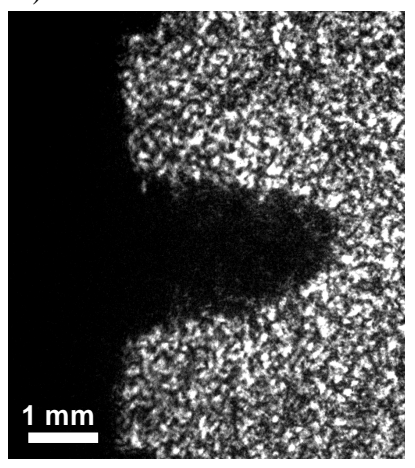

c.)

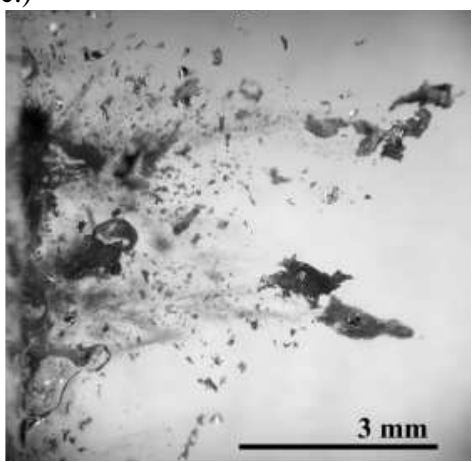

d.)

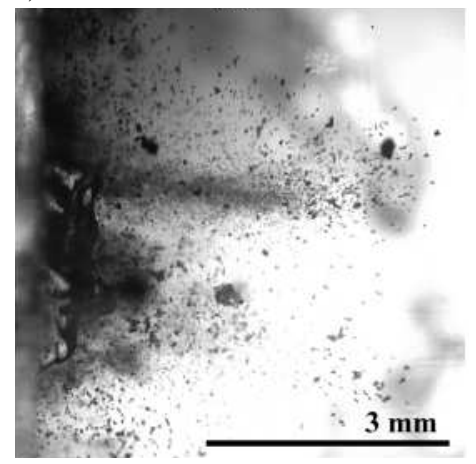

e.)

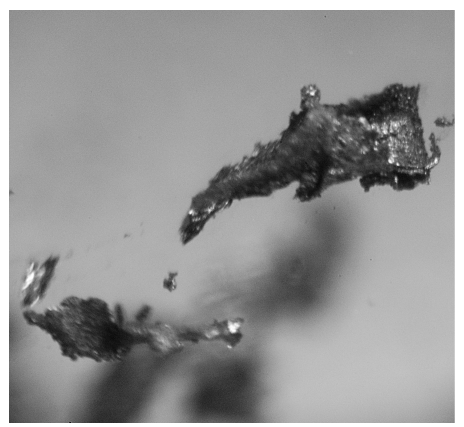

f.)

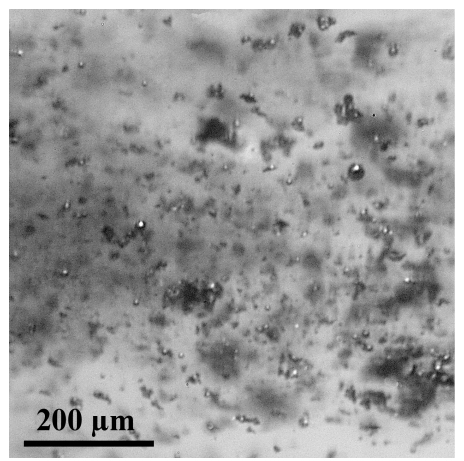

Fig. 5. (a, b) Optical transverse shadowgraphs recorded behind the free-surface of laser shock-loaded iron targets. (c, d) Optical micrographs of fragments recovered in a gel set behind the corresponding iron targets.

The impact surface is vertical, on the left. (e, f) Detailed views of pictures (c) and (d), respectively.

Soft recovery of the ejected debris has been performed in a low-density transparent gel [10] set behind the sample free surface. Overall side views through the gels (Fig. 5c-d) comfort the aforementioned assumptions concerning the nature of the debris clouds. A large number of fragments, mostly of sub-millimetric size, is observed. After the 120GPa laser shock (Test 4, Fig. 5c), few millimetric fragments can be seen down to $\sim 7 \mathrm{~mm}$ below the impact surface and should correspond to pieces of the thin $(\sim 10-20 \mu \mathrm{m}$-thick according to closer views of these fragments) first spalled layer. The presence of finer fragments closer to the impact surface of the gel seems to indicate that this first spall was followed by a cloud of smaller and slower debris, as also suggested by shadowgraph in Fig. 5a. According to their shape, these fragments were certainly created in solid state (Fig. 5e). Then they may have been ejected at a velocity with some radial, outward component 
so that small fragments can be seen over a zone much wider than the $3 \mathrm{~mm}$-diameter of the loaded spot. After the 240GPa laser shock (Test 5, Fig. 5d), a larger number of fragments of smaller size are observed. Most fragments are less than $50 \mu \mathrm{m}$ with some random shapes and rough surfaces similar to those observed in Test 4 . However, some spherical fragments of diameter ranging from $\sim 7$ to $30 \mu \mathrm{m}$ can be seen (Fig. 5f). They have probably been generated in liquid state, turned spherical due to surface tension then solidified. Thus these observations are consistent with the prediction of simulations mentioned in Section 2 and Fig. 3b. Analogous observations have been reported after laser shocks on tin $[4,5,12]$. Whether such liquid fragments come from a micro-spalling process (i.e. fragmentation upon tensile loading of molten materials near the free surface) or from deeper unspalled melted regions is difficult to infer. Nevertheless, their presence can be associated to significant amounts of melted iron ejected from the target at high velocities.

Fig. 6 shows the ejected tin debris collected in gel after Test 6 . Fragments have penetrated the gel up to $\sim 10 \mathrm{~mm}$ over a zone of $2-3 \mathrm{~mm}$-diameter in transversal directions. Most fragments are essentially spherical which indicates that they result from a fragmentation process in liquid state, namely micro-spalling. Although few solidified droplets of $\sim 100 \mu \mathrm{m}$-diameter can be seen, the majority of fragments have a size of about $10 \mu \mathrm{m}$ and probably below since smaller fragments may have not been seen due to the resolution limit of the diagnosis. These features are very similar to those observed in tin debris collected in PVC-foam for the same conditions [12,13]. Further work would include a detailed analysis of such gels to infer quantitative size distribution using highresolution (less than $1 \mu \mathrm{m}) \mathrm{X}$-Ray micro-tomography.

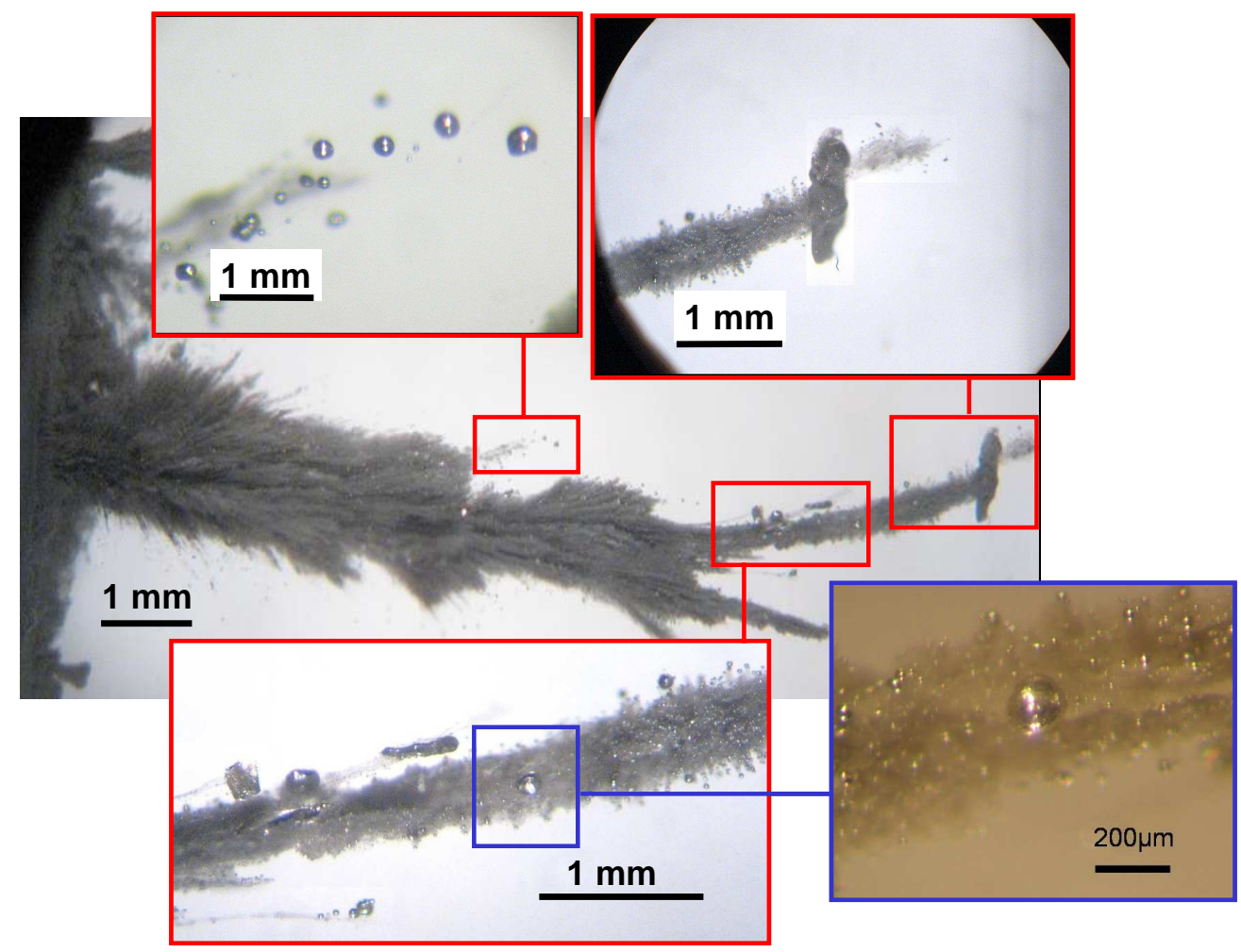

Fig. 6. Optical micrographs of fragments collected in gel set behind the free surface of laser shock-loaded tin target (Test 6). 


\section{Possible applications for modelling issues}

Experimental data discussed above are essential for developing and validating fragmentation models in dynamic conditions. In particular a key issue concerns the prediction of fragments size which may be provided by an energy-based theory [14]. The case of high temperature solid and liquid, to which this work is devoted, has been discussed more specifically by Grady (chap. 9 of [1]). For molten metals, the fragment size $s$ (its diameter in the case of spherical liquid droplet) is found to depend mainly on surface tension $\gamma$, mass density $\rho$ and dilatation rate $\dot{\varepsilon}=-\dot{\rho} / \rho$ during tensile loading (due to the reflection of the former compressive pulse upon free surface) so that $s=\left(48 \gamma / \rho \dot{\varepsilon}^{2}\right)^{1 / 3}$. This model has been applied with some success to melted tin and has been implemented in a Lagrangian wave propagation code as a failure criterion [12]. For liquid iron, with $\rho=7000 \mathrm{~kg} / \mathrm{m}^{3}$, $\gamma=2 \mathrm{~J} / \mathrm{m}^{2}$ and $\dot{\varepsilon}$ ranging from $10^{6}$ to $10^{8} \mathrm{~s}^{-1}$ (as commonly accepted in laser-shock experiments), fragment size is found to range from about one to 25 micrometers. Such prediction sounds quite realistic in regard to aforementioned observations on Test 5.

Nevertheless, such energetic models are based on rough assumptions which do not take into account the evolution of the physical mechanisms responsible for fragmentation. Micromechanical modelling approach has been initiated in order to provide some insight about this important issue in the case of liquid fragmentation [15]. The rapid expansion of an elementary hollow sphere made of compressible, viscous liquid tin has been studied in order to describe the cavitation - or void growth - process expected to be the main cause of the disintegration of melted metals.

\section{Conclusion}

Dynamic failure and fragmentation of shock-loaded metals has been investigated using different and complementary shock generators and diagnostics. Plate impact experiments allow generating wellcontrolled planar shock-wave with only a slight decay of the peak pressure through the sample. However, they usually lead to extensive destruction which strongly limits post-shock analysis. Timeresolved velocity measurements coupled to the Asay-window technique provide indirect information concerning the global kinematical properties of the debris cloud, especially in the case of microspalling where fragments are very fine. However, a comparison between measured and simulated velocity profile (not reported here, see [6]) indicates that the global - or macroscopic - expansion of the micro-spalled cloud is fairly well described by 1D simulations without capturing detailed behaviour of the ejecta.

Energy involved during laser-shock experiments are far lower so that they are less destructive, which allows to recover and to examine targets and fragments. Analysing the recovered ejecta provides an evaluation of the characteristic size of the fragments. As illustrated in this work involving a new recovery medium to collect fragments (low-density gel), but also from past studies, they are found to be micrometric, even sub-micrometric, in the case of micro-spalled tin. As for iron, fragments size are then found to range from some $10 \mu \mathrm{m}$ to some of millimetre after spall fracture in solid state, and from $\sim 7$ to $30 \mu \mathrm{m}$ after melting. In particular, the actual fragmentation process is shown to be far more complex that the idealized succession of well-defined spalled layers depicted in Fig. 1a. This may be seen as a sign of a progressive evolution of the fragmentation process from spalling to micro-spalling. Besides, the present experiments provide indirect signs of melting in high intensity shots on iron which seems consistent, at least qualitatively, with preliminary computations. 


\section{Acknowledgements}

Plate impact experiments have been conducted at the Commissariat à l'Energie Atomique (CEA) and at the Délégation Générale de l'Armement (DGA, Centre d'Etude de Gramat). The authors would like to thank P.-Y. Chanal, P.-L. Héreil, C. Prieur, F. Cinatti and C. Loupias at the DGA, as well as F. Buy, C. Voltz, F. Llorca, P. Antoine and G. Lanier at the CEA/Valduc for their essential contribution. All the staff of the LULI is greatly acknowledged for technical support during the realization of the laser-shock experiments. The access to LULI laser facilities was provided in the framework of ILP (Institut Laser Plasma, FR2707). This study was initiated and supported by the CEA-Valduc.

\section{References}

1. L. Davison, D.E. Grady, M. Shahinpoor, editors, High-Pressure Shock Compression of Solids II. Dynamic Fracture and Fragmentation (New-York, Springer-Verlag, 1996)

2. P. Andriot et al., Shock Waves in Condensed Matter (North Holland, Amsterdam, 1984)

3. D. B. Holtkamp et al., AIP Conf. Proc. 706 (2004)

4. T. De Rességuier et al., J. Appl. Phys. 101, 013506 (2007)

5. T. De Rességuier et al., Appl. Phys. Letters 92, 131910 (2008)

6. L. Signor et al., AIP Conf. Proc. 1195 (2009)

7. C. W. McCluskey et al., Rev. Sci. Instrum. 77, 113902 (2006)

8. J. R. Asay, J. Appl. Phys. 49, 12 (1978)

9. F. Buy et al., AIP Conf. Proc. 845 (2006)

10. E. Lescoute et al., Appl. Phys. Letters 95, 211905 (2009)

11. A. V. Bushman et al., Russian academy of sciences report (1992)

12. L. Signor et al., submitted to Int. J. Impact Eng. (2010)

13. T. De Resseguier et al., Int. J. Frac (2010), DOI 10.1007/s10704-009-9378-8

14. D.E. Grady, J. Mech. Phys. Solids 36, 3 (1988)

15. L. Signor et al., Arch. Mech. 60, 4 (2008) 